\title{
Anxiety and depression in women with and without chronic pelvic pain: prevalence and associated factors
}

This article was published in the following Dove Press journal: Journal of Pain Research

\author{
Vânia Meira e \\ Siqueira-Campos (1D) \\ Rosa Azevedo Da Luz $\mathbb{D D}^{2}$ \\ José Miguel de Deus $\mathbb{D D}^{2,3}$ \\ Edson Zangiacomi \\ Martinez (D) ${ }^{4}$ \\ Délio Marques Conde $\mathbb{1}^{1,3}$ \\ 'Postgraduate Program in Health \\ Sciences, Federal University of Goiás, \\ Goiânia, Goiás, Brazil; ${ }^{2}$ Teaching Hospital, \\ Women's Health Unit, Federal University \\ of Goiás, Goiânia, Goiás, Brazil; \\ ${ }^{3}$ Department of Gynecology and \\ Obstetrics, Federal University of Goiás, \\ Goiânia, Goiás, Brazil; ${ }^{4}$ Ribeirão Preto \\ Medical School, University of São Paulo, \\ Ribeirão Preto, São Paulo, Brazil
}

Objectives: To investigate the prevalence of anxiety, depression and mixed anxiety and depressive disorder (MADD) and factors associated with these conditions in women with chronic pelvic pain (CPP) compared to a pain-free control group.

Methods: A cross-sectional study was conducted with 100 women with CPP and 100 without CPP. The Hospital Anxiety and Depression Scale (HADS) was used to evaluate the presence of anxiety and depression. Sociodemographic, behavioral and clinical characteristics were investigated. Fisher's exact test was used to compare characteristics between groups. A log-binomial regression model was used, with adjustment for age, skin color, schooling, body mass index and pain. Prevalence ratios (PR), together with their 95\% confidence intervals (CI), were calculated to investigate factors associated with anxiety, depression and MADD.

Results: The prevalence of anxiety was $66 \%$ in the CPP group and $49 \%$ in the controls ( $p=0.02$ ). Depression was identified in $63 \%$ of the women with CPP and in $38 \%$ of the controls $(p<0.01)$. MADD was present in $54 \%$ of the CPP group and in $28 \%$ of the controls $(p<0.01)$. In the adjusted analysis, $\mathrm{CPP}(\mathrm{PR}=1.3 ; 95 \% \mathrm{CI}: 1.1-1.6)$, physical abuse ( $\mathrm{PR}=1.5$; 95\%CI: $1.2-1.8)$ and sexual abuse ( $\mathrm{PR}=1.5 ; 95 \% \mathrm{CI}$ : 1.1-1.8) were independently associated with anxiety. Women of 25 to 34 years of age were less likely to have anxiety ( $\mathrm{PR}=0.6 ; 95 \%$ CI: 0.4-0.8). CPP (PR=1.6; 95\%CI: 1.2-2.2), physical abuse ( $\mathrm{PR}=1.3 ; 95 \% \mathrm{CI}: 1.1-1.7)$ and sexual abuse ( $\mathrm{PR}=1.7 ; 95 \% \mathrm{CI}: 1.3-2.2)$ were independently associated with depression. $\mathrm{CPP}$ ( $\mathrm{PR}=1.9$; 95\%CI: 1.3-2.7), smoking ( $\mathrm{PR}=1.5 ; 95 \% \mathrm{CI}: 1.1-2.1)$, physical abuse $(\mathrm{PR}=1.4$; 95\%CI: $1.1-1.9)$ and sexual abuse ( $\mathrm{PR}=1.4 ; 95 \% \mathrm{CI}$ : $1.1-1.8)$ were independently associated with MADD

Conclusions: The prevalence of anxiety, depression and MADD was higher in women with CPP compared to the pain-free controls. Factors associated with mental disorders were identified. The independent association between CPP and anxiety, depression and MADD was noteworthy. These findings suggest that systematic management of psychological factors could contribute towards improving the mental health of these women.

Keywords: sexual abuse, physical abuse, age, smoking, women's health

\section{Introduction}

Chronic pain is a public health issue. However, the term chronic pain includes different types of pain such as chronic low back pain (CLBP) and chronic pelvic pain (CPP). These two distinct conditions merit particular mention in view of their negative repercussions on different areas of life. However, while CLBP consists of pain, muscle tension or stiffness below the costal margin and above the inferior
Department of Gynecology and

Obstetrics, Federal University of Goiás,

Rua 235 S/N, Setor Leste Universitário,

Goiânia, Goiás 74605-050, Brazil

Tel +55623209615I

Email delioconde@gmail.com 
gluteal folds, with or without leg pain (sciatica), persisting for more than three months, ${ }^{1}$ CPP in women can be described as noncyclic pain in the pelvic region persisting for at least six months, causing functional incapacity or requiring medical care. ${ }^{2}$ The prevalence of CPP in women varies across regions and countries. Prevalence rates of CPP have been reported as $19 \%$ in Brazil, $60.5 \%$ in China and $20 \%$ in the United States, with rates being higher in women of reproductive age. ${ }^{3-6}$

The etiology of CPP remains unclear; however, it appears to be multifactorial, involving both gynecological conditions and non-gynecological conditions such as those involving the digestive, urological, neurological and musculoskeletal systems. ${ }^{7}$ The cause of CPP remains unidentified in approximately half of the women affected. ${ }^{6,7}$ Furthermore, even when organic diseases are diagnosed, their finding appears to be rather incidental, with no epidemiological criteria pointing to the cause of pelvic pain. ${ }^{8}$

CPP could possibly be a functional somatic syndrome, since both conditions share clinical similarities such as pain constituting the principal symptom, chronicity, absence of laboratory findings, being more common in women, worsening with stress and menstruation, and being associated with depression, anxiety and sexual and physical abuse. ${ }^{8}$ In addition, CPP represents an economic burden and may be associated with negative repercussions at work, in sexual functioning and mental health. ${ }^{6,7,9,10}$ These repercussions, taken together, may exert a negative effect on quality of life. ${ }^{10,11}$ Previous studies have shown an association between CPP in women and mood disorders and a history of physical and sexual abuse..$^{3,6,12}$

Anxiety and depression are the mental disorders most commonly investigated in patients with chronic pain. Studies have shown a high frequency of psychological problems in women with CPP, although the rates vary between authors. ${ }^{10,13}$ An association has also been reported between symptoms of depression and anxiety and a history of physical and/or sexual abuse both in women with CPP and in those without CPP. ${ }^{13-18}$ This emphasizes the importance of evaluating the occurrence of such issues and its relationship with mental disorders.

In view of the increasing evidence associating a psychosocial context with CPP, the objective of the present study was to investigate the prevalence of anxiety, depression and mixed anxiety and depressive disorder (MADD) and the factors associated with these conditions in women with CPP compared to a group of controls without CPP.

\section{Patients and methods}

\section{Sample size}

Sample size was calculated based on the power to detect a statistically significant difference between two prevalence rates. Considering an expected prevalence rate of anxiety of $73 \%$ in the group with CPP, as based on a previous report, ${ }^{10}$ a sample size of 100 women in each group (in the CPP group and in the control group without CPP) would be sufficient to detect differences of approximately $20 \%$, with a power of $80 \%$ and a significance level of 0.05 . This sample size would also be sufficient to detect a difference of $20 \%$ between the groups, considering an expected prevalence rate of depression of $40 \%$, as previously described. ${ }^{10}$

\section{Participants}

A cross-sectional study was conducted between October 2014 and February 2016 at the Department of Obstetrics and Gynecology, Teaching Hospital, Federal University of Goiás, Goiânia, Goiás, Brazil. All the procedures complied with the Declaration of Helsinki. The institution's internal review board approved the study protocol and all the participants signed an informed consent form.

Participant selection has already been described in a publication resulting from a study on the quality of life of women with CPP. ${ }^{11}$ Women at least 18 years old with a complaint of CPP and who were being followed up as outpatients were invited to participate in the study. The cause of CPP was not investigated, since that was not one of the objectives of that study. The women with CPP were included in the study irrespective of whether or not they were undergoing any form of treatment for the pain. The comparison group included women of at least 18 years of age without CPP who were being seen for benign gynecological diseases such as fibroids, endometrial polyps and ovarian cysts. The exclusion criteria were being pregnant, having been pregnant in the preceding 12 months and having a history of cancer. A total of 207 consecutive women, who had been attending outpatient consultations, were invited to participate in the study, 103 with CPP and 104 pain-free controls. Three women with CPP and four pain-free women refused to participate in the study, alleging lack of time. Therefore, the final sample consisted of 100 women with CPP and 100 controls.

Certain sociodemographic, behavioral and clinical characteristics were selected for inclusion in the study based on the best evidence available in the literature on 
mental disorders in women with CPP. Data on these characteristics were collected at interviews and included: age, skin color, body mass index (BMI; $\mathrm{kg} / \mathrm{m}^{2}$ ), marital status, monthly family income (Brazilian reais, $\mathrm{R} \$$ ), schooling, employment status, parity, menopausal status, physical abuse, sexual abuse, physical activity, smoking and alcohol consumption. Hypertension and diabetes mellitus were self-reported. History of physical abuse was based on the answer to the question: "Have you ever suffered physical abuse?" (yes/no). A history of sexual abuse was established from the question: "Have you ever suffered sexual abuse?" (yes/no). Current smokers and those who had stopped smoking less than a year previously were defined as smokers, while never-smokers and those who had stopped smoking over a year ago were considered nonsmokers. Menopausal status was classified as postmenopausal following at least 12 months of amenorrhea.

In the CPP group, the duration of pain was investigated. The intensity of pelvic pain was evaluated using a $10-\mathrm{cm}(0-10$ points) visual analogue scale, with zero representing the absence of pain and 10 the worst pain imaginable.

\section{Anxiety and depression}

The Hospital Anxiety and Depression Scale (HADS) was used to identify symptoms of anxiety and depression. ${ }^{19}$ This is a 14-item self-report questionnaire, with two subscales containing seven items each, the first dealing with anxiety (HAD-A) and the second with depression (HAD-D). Each item is awarded a score of 0 to 3 , resulting in a range of 0 to 21 based on the sum of the scores obtained for each subscale. The HADS has been validated for use in

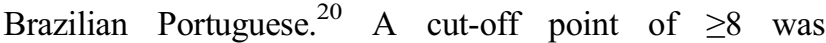
established for a diagnosis of anxiety and for depression. ${ }^{20}$

\section{Statistical analysis}

Associations between sociodemographic, behavioral and clinical variables and the outcome measures (anxiety and depression) were calculated as prevalence ratios (PR) with their respective $95 \%$ confidence intervals (CI) using logbinomial regression models. ${ }^{21}$ These models allow crude and adjusted PR to be calculated, taking into consideration the effect of possible confounders such as age, skin color, schooling, BMI and pain. When the $95 \% \mathrm{CI}$ around the PR does not include 1.0, this indicates a statistically significant association $(p<0.05)$. The entire statistical analysis was performed using the SAS statistical software program, version 9.4 (SAS Institute, Cary, NC, USA).

\section{Results}

Over half the women with CPP (54\%) and over half the controls $(56 \%)$ were under 40 years of age $(p=0.58)$. In the CPP group, $16 \%$ of the women reported smoking compared to $6 \%$ of the controls $(p=0.04)$. There was no statistically significant difference between the groups in relation to any of the other characteristics evaluated (Table 1).

The prevalence of anxiety was $66 \%$ in the group of women with CPP and $49 \%$ in the controls $(p=0.02)$. Depression was identified in $63 \%$ of the CPP group and in $38 \%$ of the controls $(p<0.01)$. MADD was present in $54 \%$ and $28 \%$ of the women in the CPP and control groups, respectively $(p<0.01)$ (Table 2$)$.

In the adjusted analysis, CPP $(\mathrm{PR}=1.3$; 95\% CI: $1.1-$ 1.6), physical abuse ( $\mathrm{PR}=1.5 ; 95 \% \mathrm{CI}: 1.2-1.8)$ and sexual abuse ( $\mathrm{PR}=1.5$; 95\% CI: 1.1-1.8) were independently associated with anxiety. Women of 25 to 34 years of age were less likely to suffer anxiety ( $\mathrm{PR}=0.6 ; 95 \% \mathrm{CI}$ : $0.4-0.8)$ (Table 3).

Following adjustment for the covariates, $\mathrm{CPP}(\mathrm{PR}=1.6$; 95\%CI: 1.2-2.2), physical abuse ( $\mathrm{PR}=1.3 ; 95 \% \mathrm{CI}: 1.1-$ $1.7)$ and sexual abuse $(\mathrm{PR}=1.7 ; 95 \% \mathrm{CI}: 1.3-2.2)$ were independently associated with depression (Table 4).

Following regression analysis, $\mathrm{CPP}(\mathrm{PR}=1.9 ; 95 \% \mathrm{CI}$ : 1.3-2.7), smoking ( $\mathrm{PR}=1.5$; 95\%CI: 1.1-2.1), physical abuse (PR=1.4; 95\%CI: 1.1-1.9) and sexual abuse $(\mathrm{PR}=1.4 ; 95 \% \mathrm{CI}: 1.1-1.8)$ were independently associated with MADD (Table 5).

The mean duration of CPP was $7.0 \pm 6.0$ years and the mean intensity of the pain was $7.8 \pm 2.1$. No statistically significant association was found between either the intensity or the duration of the pain and anxiety, depression or MADD (data not shown).

\section{Discussion}

The present study was designed to investigate the prevalence of anxiety, depression and MADD and the factors associated with these conditions in women with CPP compared to pain-free controls. The global prevalence of anxiety in women worldwide is $4.6 \%$, with a rate of $9.3 \%$ in Brazil, while the global prevalence of depression is $5.1 \%$ worldwide and $5.8 \%$ in Brazil. ${ }^{22}$ In the present study, the prevalence of anxiety in women with CPP was $66 \%$, with prevalence rates of depression and MADD of $63 \%$ and $54 \%$, respectively. These rates were significantly higher compared to those found in the control group. Despite this difference, the prevalence of mental disorders 
Table I Characteristics of the group of women with chronic pelvic pain and the pain-free control group

\begin{tabular}{|c|c|c|c|c|c|c|}
\hline \multicolumn{2}{|l|}{ Characteristics } & \multicolumn{2}{|c|}{ With pain $(n=100)$} & \multicolumn{2}{|c|}{ Without pain $(n=100)$} & \multirow[t]{2}{*}{$p$-value ${ }^{a}$} \\
\hline & & $\mathbf{n}$ & (\%) & $\mathbf{n}$ & (\%) & \\
\hline Age (years) & $\begin{array}{l}<25 \\
25-34 \\
35-39 \\
40-44 \\
\geq 45\end{array}$ & $\begin{array}{l}6 \\
29 \\
19 \\
26 \\
20\end{array}$ & $\begin{array}{l}(6) \\
(29) \\
(19) \\
(26) \\
(20)\end{array}$ & $\begin{array}{l}12 \\
26 \\
18 \\
21 \\
23\end{array}$ & $\begin{array}{l}(I 2) \\
(26) \\
(I 8) \\
(2 I) \\
(23)\end{array}$ & 0.58 \\
\hline Skin color & $\begin{array}{l}\text { White } \\
\text { Black } \\
\text { Brown } \\
\text { Yellow }\end{array}$ & $\begin{array}{l}33 \\
12 \\
53 \\
2\end{array}$ & $\begin{array}{l}(33) \\
(12) \\
(53) \\
(2)\end{array}$ & $\begin{array}{l}27 \\
10 \\
62 \\
1\end{array}$ & $\begin{array}{l}(27) \\
(10) \\
(62) \\
(1)\end{array}$ & 0.61 \\
\hline Marital status & $\begin{array}{l}\text { Single } \\
\text { Married } \\
\text { Divorced } \\
\text { Widowed }\end{array}$ & $\begin{array}{l}21 \\
67 \\
10 \\
2\end{array}$ & $\begin{array}{l}(21) \\
(67) \\
(10) \\
(2)\end{array}$ & $\begin{array}{l}30 \\
58 \\
12 \\
0\end{array}$ & $\begin{array}{l}(30) \\
(58) \\
(12) \\
0\end{array}$ & 0.22 \\
\hline Monthly family income (R\$) & $\begin{array}{l}\leq 1,000 \\
I, 00 I-1,600 \\
I, 600-2,300 \\
>2,300\end{array}$ & $\begin{array}{l}25 \\
25 \\
26 \\
24\end{array}$ & $\begin{array}{l}(25) \\
(25) \\
(26) \\
(24)\end{array}$ & $\begin{array}{l}24 \\
32 \\
18 \\
26\end{array}$ & $\begin{array}{l}(24) \\
(32) \\
(18) \\
(26)\end{array}$ & 0.50 \\
\hline Schooling & $\begin{array}{l}<I I \text { years } \\
\geq I I \text { years }\end{array}$ & $\begin{array}{l}52 \\
48\end{array}$ & $\begin{array}{l}(52) \\
(48)\end{array}$ & $\begin{array}{l}44 \\
56\end{array}$ & $\begin{array}{l}(44) \\
(56)\end{array}$ & 0.32 \\
\hline Employment status & $\begin{array}{l}\text { Employed } \\
\text { Unemployed } \\
\text { Retired } \\
\text { Homemaker }\end{array}$ & $\begin{array}{l}44 \\
7 \\
2 \\
47\end{array}$ & $\begin{array}{l}(44) \\
(7) \\
(2) \\
(47)\end{array}$ & $\begin{array}{l}54 \\
8 \\
4 \\
34\end{array}$ & $\begin{array}{l}(54) \\
(8) \\
(4) \\
(34)\end{array}$ & 0.28 \\
\hline Smoking & $\begin{array}{l}\text { Smoker } \\
\text { Nonsmoker }\end{array}$ & $\begin{array}{l}16 \\
84\end{array}$ & $\begin{array}{l}(16) \\
(84)\end{array}$ & $\begin{array}{l}6 \\
94\end{array}$ & $\begin{array}{l}(6) \\
(94)\end{array}$ & 0.04 \\
\hline Physical activity & $\begin{array}{l}\text { No } \\
\text { Yes }\end{array}$ & $\begin{array}{l}73 \\
27\end{array}$ & $\begin{array}{l}(73) \\
(27)\end{array}$ & $\begin{array}{l}81 \\
19\end{array}$ & $\begin{array}{l}(81) \\
(19)\end{array}$ & 0.24 \\
\hline Body mass index $\left(\mathrm{kg} / \mathrm{m}^{2}\right)$ & $\begin{array}{l}<25 \\
25.00-29.99 \\
\geq 30\end{array}$ & $\begin{array}{l}50 \\
35 \\
15\end{array}$ & $\begin{array}{l}(50) \\
(35) \\
(15)\end{array}$ & $\begin{array}{l}43 \\
36 \\
21\end{array}$ & $\begin{array}{l}(43) \\
(36) \\
(2 I)\end{array}$ & 0.46 \\
\hline Alcohol consumption & $\begin{array}{l}\text { No } \\
\text { Yes }\end{array}$ & $\begin{array}{l}64 \\
36\end{array}$ & $\begin{array}{l}(64) \\
(36)\end{array}$ & $\begin{array}{l}58 \\
42\end{array}$ & $\begin{array}{l}(58) \\
(42)\end{array}$ & 0.47 \\
\hline Diabetes & $\begin{array}{l}\text { No } \\
\text { Yes }\end{array}$ & $\begin{array}{l}93 \\
7\end{array}$ & $\begin{array}{l}(93) \\
(7)\end{array}$ & $\begin{array}{l}98 \\
2\end{array}$ & $\begin{array}{l}(98) \\
(2)\end{array}$ & 0.10 \\
\hline Hypertension & $\begin{array}{l}\text { No } \\
\text { Yes }\end{array}$ & $\begin{array}{l}83 \\
17\end{array}$ & $\begin{array}{l}(83) \\
(17)\end{array}$ & $\begin{array}{l}84 \\
16\end{array}$ & $\begin{array}{l}(84) \\
(16)\end{array}$ & 1.00 \\
\hline Physical abuse & $\begin{array}{l}\text { No } \\
\text { Yes }\end{array}$ & $\begin{array}{l}75 \\
25\end{array}$ & $\begin{array}{l}(75) \\
(25)\end{array}$ & $\begin{array}{l}74 \\
26\end{array}$ & $\begin{array}{l}(74) \\
(26)\end{array}$ & 1.00 \\
\hline Sexual abuse & $\begin{array}{l}\text { No } \\
\text { Yes }\end{array}$ & $\begin{array}{l}82 \\
18\end{array}$ & $\begin{array}{l}(82) \\
(18)\end{array}$ & $\begin{array}{l}89 \\
11\end{array}$ & $\begin{array}{l}(89) \\
\text { (II) }\end{array}$ & 0.23 \\
\hline Menopausal status & $\begin{array}{l}\text { Premenopausal } \\
\text { Postmenopausal }\end{array}$ & $\begin{array}{l}79 \\
21\end{array}$ & $\begin{array}{l}(79) \\
(2 I)\end{array}$ & $\begin{array}{l}79 \\
21\end{array}$ & $\begin{array}{l}(79) \\
(2 I)\end{array}$ & 1.00 \\
\hline Parity & $\begin{array}{l}\text { Nulliparous } \\
\geq 1\end{array}$ & $\begin{array}{l}14 \\
86\end{array}$ & $\begin{array}{l}(14) \\
(86)\end{array}$ & $\begin{array}{l}25 \\
75\end{array}$ & $\begin{array}{l}(25) \\
(75)\end{array}$ & 0.07 \\
\hline
\end{tabular}

Note: ${ }^{\text {a}}$ Fisher's exact test. 
Table 2 Prevalence of anxiety, depression and mixed anxiety and depressive disorder in the group of women with chronic pelvic pain and in the pain-free control group

\begin{tabular}{|c|c|c|c|c|c|c|}
\hline \multicolumn{2}{|l|}{ Variables } & \multicolumn{2}{|c|}{ With pain $(n=100)$} & \multicolumn{2}{|c|}{ Without pain $(n=100)$} & \multirow{2}{*}{$\begin{array}{l}p \text {-value }{ }^{\text {a }} \\
0.02\end{array}$} \\
\hline Anxiety & $\begin{array}{l}\text { No } \\
\text { Yes }\end{array}$ & $\begin{array}{l}\mathbf{n} \\
34 \\
66\end{array}$ & $\begin{array}{l}(\%) \\
(34) \\
(66)\end{array}$ & $\begin{array}{l}\text { n } \\
51 \\
49\end{array}$ & $\begin{array}{l}(\%) \\
(51) \\
(49)\end{array}$ & \\
\hline Depression & $\begin{array}{l}\text { No } \\
\text { Yes }\end{array}$ & $\begin{array}{l}37 \\
63\end{array}$ & $\begin{array}{l}(37) \\
(63)\end{array}$ & $\begin{array}{l}62 \\
38\end{array}$ & $\begin{array}{l}(62) \\
(38)\end{array}$ & $<0.01$ \\
\hline Anxiety and depression & Both conditions & 54 & (54) & 28 & (28) & $<0.01$ \\
\hline
\end{tabular}

Note: ${ }^{\text {a}}$ Fisher's exact test.

in the pain-free controls is noteworthy. The high frequency of mental disorders in the control group could be related to the fact that the women have benign gynecological disorders. Previous studies have shown that benign diseases such as ovarian cysts and fibroids could exert a negative impact on women's mental health, giving rise to disorders that include anxiety and depression. ${ }^{23,24}$

In a study conducted in Brazil, $73 \%$ and $40 \%$ of the women with CPP had anxiety and depression, respectively. ${ }^{10}$ In a cross-sectional study conducted in the United States involving 107 women with CPP, the prevalence of anxiety was $38.6 \%$, while the prevalence of depression was $25.7 \% .{ }^{13}$ However, other authors have reported no significant differences in anxiety levels between women with and without CPP. ${ }^{9}$ The discrepancies in results across the different studies may be due to the use of different instruments such as the Beck Anxiety Inventory and the Beck Depression Inventory and the HADS. ${ }^{9,10,25}$ In addition, the prevalence of mental disorders may differ across regions, cultures and countries. ${ }^{26}$ Another important point is that while some authors included a group without CPP for comparison, as in the present study, other investigators evaluated only women with CPP. ${ }^{9,10,13,25}$ However, analysis of the studies showed a high prevalence of anxiety and depression in women with CPP. Therefore, it is important to identify the factors associated with these conditions.

In this study, CPP was independently associated with anxiety, depression and MADD. Previous studies with women have shown an association between CPP and mental disorders such as depression, anxiety, neuroticism and somatization. ${ }^{3,12}$ In a population-based study conducted in Scotland with 2,088 women, having depression was significantly associated with CPP (odds ratio [OR] $=1.61 ; 95 \%$ CI: $1.09-2.38){ }^{6}$ In a systematic review with meta-analysis, anxiety $(\mathrm{OR}=2.28$; 99\%CI: $1.41-3.7)$ and depression $(\mathrm{OR}=2.69$; 99\%CI: $1.86-3.88)$ were associated with CPP in women. ${ }^{12}$ Depression may be present in up to one-third of patients with chronic pain. ${ }^{27}$ On the other hand, around $65 \%$ of depressed patients have some type of chronic pain. ${ }^{28}$ The present findings, taken together with the results of other studies, indicate a consistent association between chronic pain, including CPP, and mental disorders. This evidence should be taken into consideration when defining the diagnostic and therapeutic management of such disorders in women with CPP.

The present findings show that women of 25 to 34 years of age were less likely to have anxiety compared to younger women. Age can be a determining factor in anxiety symptoms. ${ }^{29}$ The prevalence of anxiety disorders increases between 10 and 19 years of age and peaks at 20 to 24 years of age. ${ }^{26}$ The reason why the $25-34$-years age group is less likely to experience symptoms of anxiety is unclear. In general, women in this age group have completed their education, are employed, married and have children. Although there is no evidence at the present moment, factors such as being employed and having already formed a family could hypothetically protect these women's mental health. If confirmed, this hypothesis could at least partially explain the results of the present study. Therefore, future studies are required to delve further into the association between age and mental diseases.

Physical and sexual abuse were found to be associated with anxiety, depression and MADD in both the unadjusted and adjusted analyses. Other studies, however, have reported conflicting findings regarding these associations. ${ }^{14-16,18,30}$ Some authors identified an association between sexual abuse and anxiety and depression. ${ }^{18,30}$ Others have described an association between physical and 
Table 3 Factors associated with anxiety in women with chronic pelvic pain and in pain-free controls

\begin{tabular}{|c|c|c|c|c|c|c|}
\hline \multicolumn{2}{|l|}{ Factors } & \multirow{3}{*}{$\begin{array}{l}\text { Total } \\
100 \\
100\end{array}$} & \multicolumn{2}{|c|}{ Anxiety } & \multirow{3}{*}{$\begin{array}{l}\text { Crude } \\
\text { PR (95\%Cl) } \\
\text { Reference } \\
1.3(1.1-1.7)^{b}\end{array}$} & \multirow{3}{*}{$\begin{array}{l}\text { Adjusted } \\
\text { PR }(95 \% \mathrm{Cl})^{\mathrm{a}} \\
\text { Reference } \\
1.3(1.1-1.6)^{\mathrm{b}}\end{array}$} \\
\hline & & & \multirow{2}{*}{$\begin{array}{l}n \\
49 \\
66\end{array}$} & \multirow{2}{*}{$\begin{array}{l}(\%) \\
(49.0) \\
(66.0)\end{array}$} & & \\
\hline Pain & $\begin{array}{l}\text { Without pain } \\
\text { With pain }\end{array}$ & & & & & \\
\hline Age (years) & $\begin{array}{l}<25 \\
25-34 \\
35-39 \\
40-44 \\
\geq 45\end{array}$ & $\begin{array}{l}18 \\
55 \\
37 \\
47 \\
43\end{array}$ & $\begin{array}{l}14 \\
26 \\
22 \\
26 \\
27\end{array}$ & $\begin{array}{l}(77.8) \\
(47.3) \\
(59.5) \\
(55.3) \\
(62.8)\end{array}$ & $\begin{array}{l}\text { Reference } \\
0.6(0.4-0.9)^{\mathrm{b}} \\
0.7(0.5-1.1) \\
0.7(0.4-1.1) \\
0.8(0.5-1.1)\end{array}$ & $\begin{array}{l}\text { Reference } \\
0.6(0.4-0.8)^{\mathrm{b}} \\
0.7(0.4-1.0) \\
0.7(0.4-1.0) \\
0.7(0.5-1.2)\end{array}$ \\
\hline Skin color & $\begin{array}{l}\text { White } \\
\text { Black } \\
\text { Brown } \\
\text { Yellow }\end{array}$ & $\begin{array}{l}60 \\
22 \\
115 \\
3\end{array}$ & $\begin{array}{l}33 \\
15 \\
66 \\
1\end{array}$ & $\begin{array}{l}(55.0) \\
(68.2) \\
(57.4) \\
(33.3)\end{array}$ & $\begin{array}{l}\text { Reference } \\
1.2(0.8-1.8) \\
1.0(0.7-1.4) \\
0.6(0.1-3.1)\end{array}$ & $\begin{array}{l}\text { Reference } \\
1.2(0.8-1.8) \\
1.0(0.7-1.3) \\
0.6(0.1-2.3)\end{array}$ \\
\hline Marital status & $\begin{array}{l}\text { Single } \\
\text { Married } \\
\text { Divorced } \\
\text { Widowed }\end{array}$ & $\begin{array}{l}51 \\
125 \\
22 \\
2\end{array}$ & $\begin{array}{l}25 \\
73 \\
15 \\
2\end{array}$ & $\begin{array}{l}(49.0) \\
(58.4) \\
(68.2) \\
(100.0)\end{array}$ & $\begin{array}{l}\text { Reference } \\
1.1(0.8-1.4) \\
1.2(0.9-1.7) \\
-\end{array}$ & $\begin{array}{l}\text { Reference } \\
1.1(0.8-1.6) \\
1.2(0.8-1.9) \\
-\end{array}$ \\
\hline Monthly family income (R\$) & $\begin{array}{l}\leq 1,000 \\
I, 001-1,600 \\
I, 600-2,300 \\
>2,300\end{array}$ & $\begin{array}{l}49 \\
57 \\
44 \\
50\end{array}$ & $\begin{array}{l}29 \\
31 \\
29 \\
26\end{array}$ & $\begin{array}{l}(59.2) \\
(54.4) \\
(65.9) \\
(52.0)\end{array}$ & $\begin{array}{l}\text { Reference } \\
0.9(0.6-1.3) \\
1.1(0.8-1.5) \\
0.8(0.6-1.3)\end{array}$ & $\begin{array}{l}\text { Reference } \\
0.9(0.7-1.2) \\
1.0(0.7-1.3) \\
0.8(0.6-1.2)\end{array}$ \\
\hline Employment status & $\begin{array}{l}\text { Employed } \\
\text { Unemployed } \\
\text { Retired } \\
\text { Homemaker }\end{array}$ & $\begin{array}{l}98 \\
15 \\
6 \\
81\end{array}$ & $\begin{array}{l}49 \\
11 \\
3 \\
52\end{array}$ & $\begin{array}{l}(50.0) \\
(73.3) \\
(50.0) \\
(64.2)\end{array}$ & $\begin{array}{l}\text { Reference } \\
1.5(1.1-2.1)^{\mathrm{b}} \\
\mathrm{I} .0(0.4-2.3) \\
\mathrm{I} .3(0.9-1.7)\end{array}$ & $\begin{array}{l}\text { Reference } \\
1.2(0.8-1.6) \\
1.0(0.6-1.6) \\
1.1(0.9-1.3)\end{array}$ \\
\hline Schooling & $\begin{array}{l}<11 \text { years } \\
\geq 11 \text { years }\end{array}$ & $\begin{array}{l}96 \\
104\end{array}$ & $\begin{array}{l}56 \\
59\end{array}$ & $\begin{array}{l}(58.3) \\
(56.7)\end{array}$ & $\begin{array}{l}\text { Reference } \\
0.9(0.7-1.2)\end{array}$ & $\begin{array}{l}\text { Reference } \\
\text { I.0 (0.7-I.3) }\end{array}$ \\
\hline Smoking & $\begin{array}{l}\text { Nonsmoker } \\
\text { Smoker }\end{array}$ & $\begin{array}{l}178 \\
22\end{array}$ & $\begin{array}{l}98 \\
17\end{array}$ & $\begin{array}{l}(55.1) \\
(77.3)\end{array}$ & $\begin{array}{l}\text { Reference } \\
1.4(1.1-1.8)^{b}\end{array}$ & $\begin{array}{l}\text { Reference } \\
\text { I.I (0.8-I.4) }\end{array}$ \\
\hline Physical activity & $\begin{array}{l}\text { Yes } \\
\text { No }\end{array}$ & $\begin{array}{l}46 \\
154\end{array}$ & $\begin{array}{l}23 \\
92\end{array}$ & $\begin{array}{l}(50.0) \\
(59.7)\end{array}$ & $\begin{array}{l}\text { Reference } \\
\text { I.2 (0.8-1.6) }\end{array}$ & $\begin{array}{l}\text { Reference } \\
\text { I.2(0.8-I.5) }\end{array}$ \\
\hline Body mass index $\left(\mathrm{kg} / \mathrm{m}^{2}\right)$ & $\begin{array}{l}<25 \\
25.00-29.99 \\
\geq 30\end{array}$ & $\begin{array}{l}93 \\
71 \\
36\end{array}$ & $\begin{array}{l}52 \\
40 \\
23\end{array}$ & $\begin{array}{l}(55.9) \\
(56.3) \\
(63.9)\end{array}$ & $\begin{array}{l}\text { Reference } \\
\text { I.0 (0.7-I.3) } \\
\text { I.I (0.8-I.5) }\end{array}$ & $\begin{array}{l}\text { Reference } \\
\text { I.0 (0.7-I.4) } \\
\text { I.2(0.8-I.7) }\end{array}$ \\
\hline Alcohol consumption & $\begin{array}{l}\text { No } \\
\text { Yes }\end{array}$ & $\begin{array}{l}122 \\
78\end{array}$ & $\begin{array}{l}71 \\
44\end{array}$ & $\begin{array}{l}(58.2) \\
(56.4)\end{array}$ & $\begin{array}{l}\text { Reference } \\
\text { I.0 (0.8-I.3) }\end{array}$ & $\begin{array}{l}\text { Reference } \\
\text { I.0 (0.7-I.4) }\end{array}$ \\
\hline Diabetes & $\begin{array}{l}\text { No } \\
\text { Yes }\end{array}$ & $\begin{array}{l}191 \\
9\end{array}$ & $\begin{array}{l}108 \\
7\end{array}$ & $\begin{array}{l}(56.5) \\
(77.8)\end{array}$ & $\begin{array}{l}\text { Reference } \\
\text { I.4 (0.9-2.0) }\end{array}$ & $\begin{array}{l}\text { Reference } \\
\text { I.I (0.6-I.8) }\end{array}$ \\
\hline Hypertension & $\begin{array}{l}\text { No } \\
\text { Yes }\end{array}$ & $\begin{array}{l}167 \\
33\end{array}$ & $\begin{array}{l}95 \\
20\end{array}$ & $\begin{array}{l}(56.9) \\
(60.6)\end{array}$ & $\begin{array}{l}\text { Reference } \\
\text { I.I (0.7-I.5) }\end{array}$ & $\begin{array}{l}\text { Reference } \\
\text { I.0 (0.7-I.5) }\end{array}$ \\
\hline Physical abuse & $\begin{array}{l}\text { No } \\
\text { Yes }\end{array}$ & $\begin{array}{l}149 \\
51\end{array}$ & $\begin{array}{l}76 \\
39\end{array}$ & $\begin{array}{l}(51.0) \\
(76.5)\end{array}$ & $\begin{array}{l}\text { Reference } \\
1.5(1.2-1.9)^{\mathrm{b}}\end{array}$ & $\begin{array}{l}\text { Reference } \\
1.5(1.2-1.8)^{\mathrm{b}}\end{array}$ \\
\hline Sexual abuse & $\begin{array}{l}\text { No } \\
\text { Yes }\end{array}$ & $\begin{array}{l}171 \\
29\end{array}$ & $\begin{array}{l}91 \\
24\end{array}$ & $\begin{array}{l}(53.2) \\
(82.8)\end{array}$ & $\begin{array}{l}\text { Reference } \\
1.6(1.2-1.9)^{b}\end{array}$ & $\begin{array}{l}\text { Reference } \\
1.5(1 .|-| .8)^{b}\end{array}$ \\
\hline Menopausal status & $\begin{array}{l}\text { Premenopausal } \\
\text { Postmenopausal }\end{array}$ & $\begin{array}{l}158 \\
42\end{array}$ & $\begin{array}{l}91 \\
24\end{array}$ & $\begin{array}{l}(57.6) \\
(57.1)\end{array}$ & $\begin{array}{l}\text { Reference } \\
\text { I.0 (0.7-I.3) }\end{array}$ & $\begin{array}{l}\text { Reference } \\
\text { I.0 (0.7-I.4) }\end{array}$ \\
\hline Parity & $\begin{array}{l}\text { Nulliparous } \\
\geq 1\end{array}$ & $\begin{array}{l}39 \\
161\end{array}$ & $\begin{array}{l}20 \\
95\end{array}$ & $\begin{array}{l}(51.3) \\
(59.0)\end{array}$ & $\begin{array}{l}\text { Reference } \\
\text { I.2 (0.8-1.6) }\end{array}$ & $\begin{array}{l}\text { Reference } \\
\text { I.2 (0.7-।.9) }\end{array}$ \\
\hline
\end{tabular}

Notes: ${ }^{a}$ Prevalence ratio adjusted for age, skin color, schooling, body mass index and pain. ${ }^{b}$ Significant association $(p<0.05)$. 
Table 4 Factors associated with depression in women with chronic pelvic pain and in pain-free controls

\begin{tabular}{|c|c|c|c|c|c|c|}
\hline \multicolumn{2}{|l|}{ Factors } & \multirow{3}{*}{$\begin{array}{l}\text { Total } \\
100 \\
100\end{array}$} & \multicolumn{2}{|c|}{ Depression } & \multirow{3}{*}{$\begin{array}{l}\text { Crude } \\
\text { PR (95\%Cl) } \\
\text { Reference } \\
1.7(1.2-2.2)^{b}\end{array}$} & \multirow{3}{*}{$\begin{array}{l}\text { Adjusted } \\
\text { PR }(95 \% \mathrm{CI})^{\mathrm{a}} \\
\text { Reference } \\
\text { I.6 (I.2-2.2) }\end{array}$} \\
\hline & & & $\mathbf{n}$ & (\%) & & \\
\hline Pain & $\begin{array}{l}\text { Without pain } \\
\text { With pain }\end{array}$ & & $\begin{array}{l}38 \\
63\end{array}$ & $\begin{array}{l}(38.0) \\
(63.0)\end{array}$ & & \\
\hline Age (years) & $\begin{array}{l}<25 \\
25-34 \\
35-39 \\
40-44 \\
\geq 45\end{array}$ & $\begin{array}{l}18 \\
55 \\
37 \\
47 \\
43\end{array}$ & $\begin{array}{l}7 \\
26 \\
20 \\
25 \\
23\end{array}$ & $\begin{array}{l}(38.9) \\
(47.3) \\
(54.1) \\
(53.2) \\
(53.5)\end{array}$ & $\begin{array}{l}\text { Reference } \\
\mathrm{I} .2(0.6-2.3) \\
\mathrm{I} .4(0.7-2.7) \\
\mathrm{I} .4(0.7-2.6) \\
\mathrm{I} .4(0.7-2.6)\end{array}$ & $\begin{array}{l}\text { Reference } \\
\text { I.0 (0.5-I.9) } \\
\text { I.2 (0.6-2.2) } \\
\text { I.I }(0.6-2.2) \\
\text { I.I }(0.6-2.2)\end{array}$ \\
\hline Skin color & $\begin{array}{l}\text { White } \\
\text { Black } \\
\text { Brown } \\
\text { Yellow }\end{array}$ & $\begin{array}{l}60 \\
22 \\
115 \\
3\end{array}$ & $\begin{array}{l}29 \\
13 \\
57 \\
2\end{array}$ & $\begin{array}{l}(48.3) \\
(59.1) \\
(49.6) \\
(66.7)\end{array}$ & $\begin{array}{l}\text { Reference } \\
\text { I.2(0.7-I.9) } \\
\text { I.0 (0.7-I.4) } \\
\text { I.4 (0.5-3.2) }\end{array}$ & $\begin{array}{l}\text { Reference } \\
1.2(0.7-1.9) \\
1.0(0.7-1.3) \\
1.3(0.4-3.6)\end{array}$ \\
\hline Marital status & $\begin{array}{l}\text { Single } \\
\text { Married } \\
\text { Divorced } \\
\text { Widowed }\end{array}$ & $\begin{array}{l}51 \\
125 \\
22 \\
2\end{array}$ & $\begin{array}{l}21 \\
64 \\
15 \\
1\end{array}$ & $\begin{array}{l}(41.2) \\
(51.2) \\
(68.2) \\
(50.0)\end{array}$ & $\begin{array}{l}\text { Reference } \\
1.2(0.8-1.8) \\
1.7(1.1-2.6)^{\mathrm{b}} \\
1.2(0.2-5.0)\end{array}$ & $\begin{array}{l}\text { Reference } \\
\text { I.I }(0.7-1.6) \\
1.3(0.8-2.1) \\
0.9(0.2-3.8)\end{array}$ \\
\hline Monthly family income $(R \$)$ & $\begin{array}{l}1,000 \text { or less } \\
1,001-1,600 \\
1,600-2,300 \\
>2,300\end{array}$ & $\begin{array}{l}49 \\
57 \\
44 \\
50\end{array}$ & $\begin{array}{l}29 \\
28 \\
22 \\
22\end{array}$ & $\begin{array}{l}(59.2) \\
(49.1) \\
(50.0) \\
(44.0)\end{array}$ & $\begin{array}{l}\text { Reference } \\
0.8(0.5-1.2) \\
0.8(0.5-1.2) \\
0.7(0.5-1.1)\end{array}$ & $\begin{array}{l}\text { Reference } \\
0.8(0.5-1.2) \\
0.8(0.5-1.1) \\
0.8(0.5-1.2)\end{array}$ \\
\hline Employment status & $\begin{array}{l}\text { Employed } \\
\text { Unemployed } \\
\text { Retired } \\
\text { Homemaker }\end{array}$ & $\begin{array}{l}98 \\
15 \\
6 \\
81\end{array}$ & $\begin{array}{l}43 \\
8 \\
3 \\
47\end{array}$ & $\begin{array}{l}(43.9) \\
(53.3) \\
(50.0) \\
(58.0)\end{array}$ & $\begin{array}{l}\text { Reference } \\
1.2(0.7-2.1) \\
1.1(0.4-2.6) \\
1.3(0.9-1.8)\end{array}$ & $\begin{array}{l}\text { Reference } \\
\text { I.4 (0.8-2.5) } \\
\text { I.3 (0.5-3.0) } \\
\text { I.2(0.9-1.7) }\end{array}$ \\
\hline Schooling & $\begin{array}{l}<11 \text { years } \\
\geq 11 \text { years }\end{array}$ & $\begin{array}{l}96 \\
104\end{array}$ & $\begin{array}{l}55 \\
46\end{array}$ & $\begin{array}{l}(57.3) \\
(44.2)\end{array}$ & $\begin{array}{l}\text { Reference } \\
0.8(0.5-1.1)\end{array}$ & $\begin{array}{l}\text { Reference } \\
0.9(0.6-1.2)\end{array}$ \\
\hline Smoking & $\begin{array}{l}\text { Nonsmoker } \\
\text { Smoker }\end{array}$ & $\begin{array}{l}178 \\
22\end{array}$ & $\begin{array}{l}83 \\
18\end{array}$ & $\begin{array}{l}(46.6) \\
(8 I .8)\end{array}$ & $\begin{array}{l}\text { Reference } \\
\text { I.7 }(1.3-2.3)^{\mathrm{b}}\end{array}$ & $\begin{array}{l}\text { Reference } \\
\text { I.I (0.8-I.4) }\end{array}$ \\
\hline Physical activity & $\begin{array}{l}\text { Yes } \\
\text { No }\end{array}$ & $\begin{array}{l}46 \\
154\end{array}$ & $\begin{array}{l}17 \\
84\end{array}$ & $\begin{array}{l}(37.0) \\
(54.6)\end{array}$ & $\begin{array}{l}\text { Reference } \\
\text { I.5 (0.9-2.2) }\end{array}$ & $\begin{array}{l}\text { Reference } \\
\text { I.5 (0.9-2.2) }\end{array}$ \\
\hline Body mass index $\left(\mathrm{kg} / \mathrm{m}^{2}\right)$ & $\begin{array}{l}<25 \\
25.00-29.99 \\
\geq 30\end{array}$ & $\begin{array}{l}93 \\
71 \\
36\end{array}$ & $\begin{array}{l}47 \\
33 \\
21\end{array}$ & $\begin{array}{l}(50.5) \\
(46.5) \\
(58.3)\end{array}$ & $\begin{array}{l}\text { Reference } \\
0.9(0.6-1.3) \\
\text { I.2(0.8-I.6) }\end{array}$ & $\begin{array}{l}\text { Reference } \\
0.8(0.6-1.2) \\
\text { I.2(0.8-I.8) }\end{array}$ \\
\hline Alcohol consumption & $\begin{array}{l}\text { No } \\
\text { Yes }\end{array}$ & $\begin{array}{l}122 \\
78\end{array}$ & $\begin{array}{l}67 \\
34\end{array}$ & $\begin{array}{l}(54.9) \\
(43.6)\end{array}$ & $\begin{array}{l}\text { Reference } \\
1.3(0.9-1.7)\end{array}$ & $\begin{array}{l}\text { Reference } \\
1.2(0.8-1.6)\end{array}$ \\
\hline Diabetes & $\begin{array}{l}\text { No } \\
\text { Yes }\end{array}$ & $\begin{array}{l}191 \\
9\end{array}$ & $\begin{array}{l}93 \\
8\end{array}$ & $\begin{array}{l}(48.7) \\
(88.9)\end{array}$ & $\begin{array}{l}\text { Reference } \\
1.8(1.3-2.4)^{\mathrm{b}}\end{array}$ & $\begin{array}{l}\text { Reference } \\
1.3(0.6-2.6)\end{array}$ \\
\hline Hypertension & $\begin{array}{l}\text { No } \\
\text { Yes }\end{array}$ & $\begin{array}{l}167 \\
33\end{array}$ & $\begin{array}{l}83 \\
18\end{array}$ & $\begin{array}{l}(49.7) \\
(54.6)\end{array}$ & $\begin{array}{l}\text { Reference } \\
\text { I.I }(0.7-1.6)\end{array}$ & $\begin{array}{l}\text { Reference } \\
1.0(0.6-1.5)\end{array}$ \\
\hline Physical abuse & $\begin{array}{l}\text { No } \\
\text { Yes }\end{array}$ & $\begin{array}{l}149 \\
51\end{array}$ & $\begin{array}{l}69 \\
32\end{array}$ & $\begin{array}{l}(46.3) \\
(62.8)\end{array}$ & $\begin{array}{l}\text { Reference } \\
1.4(1.1-1.8)^{b}\end{array}$ & $\begin{array}{l}\text { Reference } \\
\text { I.3 (I.I-I.7) }\end{array}$ \\
\hline Sexual abuse & $\begin{array}{l}\text { No } \\
\text { Yes }\end{array}$ & $\begin{array}{l}171 \\
29\end{array}$ & $\begin{array}{l}76 \\
25\end{array}$ & $\begin{array}{l}(44.4) \\
(86.2)\end{array}$ & $\begin{array}{l}\text { Reference } \\
1.9(1.5-2.4)^{\mathrm{b}}\end{array}$ & $\begin{array}{l}\text { Reference } \\
\text { I.7 (I.3-2.2) }\end{array}$ \\
\hline Menopausal status & $\begin{array}{l}\text { Premenopausal } \\
\text { Postmenopausal }\end{array}$ & $\begin{array}{l}158 \\
42\end{array}$ & $\begin{array}{l}81 \\
20\end{array}$ & $\begin{array}{l}(51.3) \\
(47.6)\end{array}$ & $\begin{array}{l}\text { Reference } \\
0.9(0.6-1.3)\end{array}$ & $\begin{array}{l}\text { Reference } \\
\text { I.I (0.7-I.7) }\end{array}$ \\
\hline Parity & $\begin{array}{l}\text { Nulliparous } \\
\geq 1\end{array}$ & $\begin{array}{l}39 \\
161\end{array}$ & $\begin{array}{l}16 \\
85\end{array}$ & $\begin{array}{l}(41.0) \\
(52.8)\end{array}$ & $\begin{array}{l}\text { Reference } \\
1.3(0.8-1.9)\end{array}$ & $\begin{array}{l}\text { Reference } \\
1.0(0.6-1.6)\end{array}$ \\
\hline
\end{tabular}

Notes: ${ }^{2}$ Prevalence ratio adjusted for age, skin color, schooling, body mass index and pain. ${ }^{b}$ Significant association $(p<0.05)$. 
Table 5 Factors associated with mixed anxiety and depressive disorder in women with chronic pelvic pain and in pain-free controls

\begin{tabular}{|c|c|c|c|c|c|c|}
\hline \multicolumn{2}{|l|}{ Factors } & \multirow{3}{*}{$\begin{array}{l}\text { Total } \\
100 \\
100\end{array}$} & \multicolumn{2}{|c|}{ Mixed anxiety and depression } & \multirow{3}{*}{$\begin{array}{l}\text { Crude } \\
\text { PR (95\%Cl) } \\
\text { Reference } \\
1.9(1.3-2.8)^{b}\end{array}$} & \multirow{3}{*}{$\begin{array}{l}\text { Adjusted } \\
\text { PR }(95 \% \mathrm{CI})^{\mathrm{a}} \\
\text { Reference } \\
1.9(1.3-2.7)^{\mathrm{b}}\end{array}$} \\
\hline & & & $\mathbf{n}$ & (\%) & & \\
\hline Pain & $\begin{array}{l}\text { Without pain } \\
\text { With pain }\end{array}$ & & $\begin{array}{l}28 \\
54\end{array}$ & $\begin{array}{l}(28.0) \\
(54.0)\end{array}$ & & \\
\hline Age (years) & $\begin{array}{l}<25 \\
25-34 \\
35-39 \\
40-44 \\
\geq 45\end{array}$ & $\begin{array}{l}18 \\
55 \\
37 \\
47 \\
43\end{array}$ & $\begin{array}{l}6 \\
20 \\
17 \\
20 \\
19\end{array}$ & $\begin{array}{l}(33.3) \\
(36.4) \\
(46.0) \\
(42.6) \\
(44.2)\end{array}$ & $\begin{array}{l}\text { Reference } \\
\text { I.I }(0.5-2.3) \\
\text { I.4 }(0.6-2.9) \\
\text { I.3 }(0.6-2.7) \\
\text { I.3 }(0.6-2.8)\end{array}$ & $\begin{array}{l}\text { Reference } \\
0.9(0.4-1.9) \\
\text { I.I }(0.5-2.3) \\
\text { I.I }(0.5-2.3) \\
\text { I. } 2(0.5-2.6)\end{array}$ \\
\hline Skin color & $\begin{array}{l}\text { White } \\
\text { Black } \\
\text { Brown } \\
\text { Yellow }\end{array}$ & $\begin{array}{l}60 \\
22 \\
115 \\
3\end{array}$ & $\begin{array}{l}23 \\
11 \\
47 \\
1\end{array}$ & $\begin{array}{l}(38.3) \\
(50.0) \\
(40.9) \\
(33.3)\end{array}$ & $\begin{array}{l}\text { Reference } \\
\text { I.3 }(0.7-2.2) \\
\text { I.I }(0.7-1.6) \\
0.8(0.1-4.4)\end{array}$ & $\begin{array}{l}\text { Reference } \\
1.4(0.8-2.4) \\
1.0(0.6-1.5) \\
0.6(0.1-3.2)\end{array}$ \\
\hline Marital status & $\begin{array}{l}\text { Single } \\
\text { Married } \\
\text { Divorced } \\
\text { Widowed }\end{array}$ & $\begin{array}{l}51 \\
125 \\
22 \\
2\end{array}$ & $\begin{array}{l}16 \\
53 \\
12 \\
1\end{array}$ & $\begin{array}{l}(31.4) \\
(42.4) \\
(54.6) \\
(50.0)\end{array}$ & $\begin{array}{l}\text { Reference } \\
1.4(0.8-2.1) \\
1.7(0.9-3.0) \\
1.6(0.3-6.7)\end{array}$ & $\begin{array}{l}\text { Reference } \\
1.2(0.7-2.0) \\
1.4(0.8-2.5) \\
1.3(0.2-6.4)\end{array}$ \\
\hline Monthly family income ( $R \$)$ & $\begin{array}{l}1,000 \text { or less } \\
1,001-1,600 \\
1,600-2,300 \\
>2,300\end{array}$ & $\begin{array}{l}49 \\
57 \\
44 \\
50\end{array}$ & $\begin{array}{l}24 \\
20 \\
19 \\
19\end{array}$ & $\begin{array}{l}(49.0) \\
(35.1) \\
(43.2) \\
(38.0)\end{array}$ & $\begin{array}{l}\text { Reference } \\
0.7(0.4-1.1) \\
0.8(0.5-1.4) \\
0.7(0.4-1.2)\end{array}$ & $\begin{array}{l}\text { Reference } \\
0.7(0.4-1.1) \\
0.7(0.4-1.2) \\
0.8(0.4-1.2)\end{array}$ \\
\hline Employment status & $\begin{array}{l}\text { Employed } \\
\text { Unemployed } \\
\text { Retired } \\
\text { Homemaker }\end{array}$ & $\begin{array}{l}98 \\
15 \\
6 \\
81\end{array}$ & $\begin{array}{l}33 \\
8 \\
3 \\
38\end{array}$ & $\begin{array}{l}(33.7) \\
(53.3) \\
(50.0) \\
(46.9)\end{array}$ & $\begin{array}{l}\text { Reference } \\
1.6(0.9-2.7) \\
1.5(0.6-3.5) \\
1.4(0.9-2.0)\end{array}$ & $\begin{array}{l}\text { Reference } \\
1.8(0.9-3.3) \\
1.6(0.6-4.0) \\
1.3(0.8-1.8)\end{array}$ \\
\hline Schooling & $\begin{array}{l}<11 \text { years } \\
\geq 11 \text { years }\end{array}$ & $\begin{array}{l}96 \\
104\end{array}$ & $\begin{array}{l}43 \\
39\end{array}$ & $\begin{array}{l}(44.8) \\
(37.5)\end{array}$ & $\begin{array}{l}\text { Reference } \\
0.8(0.6-1.2)\end{array}$ & $\begin{array}{l}\text { Reference } \\
1.0(0.6-1.5)\end{array}$ \\
\hline Smoking & $\begin{array}{l}\text { Nonsmoker } \\
\text { Smoker }\end{array}$ & $\begin{array}{l}178 \\
22\end{array}$ & $\begin{array}{l}66 \\
16\end{array}$ & $\begin{array}{l}(37.1) \\
(72.7)\end{array}$ & $\begin{array}{l}\text { Reference } \\
2.0(1.4-2.7)^{\mathrm{b}}\end{array}$ & $\begin{array}{l}\text { Reference } \\
\text { I.5 (I.I-2.I) }\end{array}$ \\
\hline Physical activity & $\begin{array}{l}\text { Yes } \\
\text { No }\end{array}$ & $\begin{array}{l}46 \\
154\end{array}$ & $\begin{array}{l}14 \\
68\end{array}$ & $\begin{array}{l}(30.4) \\
(44.2)\end{array}$ & $\begin{array}{l}\text { Reference } \\
\text { I.5 (0.9-2.3) }\end{array}$ & $\begin{array}{l}\text { Reference } \\
1.5(0.9-2.4)\end{array}$ \\
\hline Body mass index $\left(\mathrm{kg} / \mathrm{m}^{2}\right)$ & $\begin{array}{l}<25 \\
25.00-29.99 \\
\geq 30\end{array}$ & $\begin{array}{l}93 \\
71 \\
36\end{array}$ & $\begin{array}{l}40 \\
25 \\
17\end{array}$ & $\begin{array}{l}(43.0) \\
(35.2) \\
(47.2)\end{array}$ & $\begin{array}{l}\text { Reference } \\
0.8(0.5-1.2) \\
\text { I.I (0.7-I.7) }\end{array}$ & $\begin{array}{l}\text { Reference } \\
0.7(0.5-I . I) \\
\text { I.2(0.7-I.8) }\end{array}$ \\
\hline Alcohol consumption & $\begin{array}{l}\text { No } \\
\text { Yes }\end{array}$ & $\begin{array}{l}122 \\
78\end{array}$ & $\begin{array}{l}53 \\
29\end{array}$ & $\begin{array}{l}(43.4) \\
(37.2)\end{array}$ & $\begin{array}{l}\text { Reference } \\
1.2(0.8-1.7)\end{array}$ & $\begin{array}{l}\text { Reference } \\
\text { I.I }(0.7-1.5)\end{array}$ \\
\hline Diabetes & $\begin{array}{l}\text { No } \\
\text { Yes }\end{array}$ & $\begin{array}{l}191 \\
9\end{array}$ & $\begin{array}{l}75 \\
7\end{array}$ & $\begin{array}{l}(39.3) \\
(77.8)\end{array}$ & $\begin{array}{l}\text { Reference } \\
2.0(1.3-2.9)^{\mathrm{b}}\end{array}$ & $\begin{array}{l}\text { Reference } \\
1.9(0.8-4.6)\end{array}$ \\
\hline Hypertension & $\begin{array}{l}\text { No } \\
\text { Yes }\end{array}$ & $\begin{array}{l}167 \\
33\end{array}$ & $\begin{array}{l}67 \\
15\end{array}$ & $\begin{array}{l}(40.1) \\
(45.5)\end{array}$ & $\begin{array}{l}\text { Reference } \\
\text { I.I (0.7-I.7) }\end{array}$ & $\begin{array}{l}\text { Reference } \\
0.9(0.5-1.5)\end{array}$ \\
\hline Physical abuse & $\begin{array}{l}\text { No } \\
\text { Yes }\end{array}$ & $\begin{array}{l}149 \\
51\end{array}$ & $\begin{array}{l}51 \\
31\end{array}$ & $\begin{array}{l}(34.2) \\
(60.8)\end{array}$ & $\begin{array}{l}\text { Reference } \\
1.8(1.2-2.4)^{\mathrm{b}}\end{array}$ & $\begin{array}{l}\text { Reference } \\
1.4(1.1-1.9)^{\mathrm{b}}\end{array}$ \\
\hline Sexual abuse & $\begin{array}{l}\text { No } \\
\text { Yes }\end{array}$ & $\begin{array}{l}17 \mid \\
29\end{array}$ & $\begin{array}{l}59 \\
23\end{array}$ & $\begin{array}{l}(34.5) \\
(79.3)\end{array}$ & $\begin{array}{l}\text { Reference } \\
1.3(1.7-3.0)^{\mathrm{b}}\end{array}$ & $\begin{array}{l}\text { Reference } \\
1.4(1.1-1.8)^{b}\end{array}$ \\
\hline Menopausal status & $\begin{array}{l}\text { Premenopausal } \\
\text { Postmenopausal }\end{array}$ & $\begin{array}{l}158 \\
42\end{array}$ & $\begin{array}{l}65 \\
17\end{array}$ & $\begin{array}{l}(4 I .1) \\
(40.5)\end{array}$ & $\begin{array}{l}\text { Reference } \\
0.9(0.6-1.5)\end{array}$ & $\begin{array}{l}\text { Reference } \\
1.0(0.6-1.6)\end{array}$ \\
\hline Parity & $\begin{array}{l}\text { Nulliparous } \\
\geq 1\end{array}$ & $\begin{array}{l}39 \\
161\end{array}$ & $\begin{array}{l}13 \\
69\end{array}$ & $\begin{array}{l}(33.3) \\
(42.9)\end{array}$ & $\begin{array}{l}\text { Reference } \\
\text { I.3 }(0.7-2.1)\end{array}$ & $\begin{array}{l}\text { Reference } \\
1.0(0.5-1.7)\end{array}$ \\
\hline
\end{tabular}

Notes: ${ }^{\text {P}}$ Prevalence ratio adjusted for age, skin color, schooling, body mass index and pain. ${ }^{b}$ Significant association $(p<0.05)$. 
sexual abuse and impaired mental health. ${ }^{14,15}$ In a crosssectional study conducted in the United States involving 273 women with CPP, a history of physical or sexual abuse in childhood, adolescence or in adult life was significantly associated with symptoms of depression. ${ }^{14}$ In another study, conducted in Australia with 230 pain-free women in general practice clinics, adult sexual violence was significantly associated with anxiety, however not with depression, following adjustment for childhood sexual abuse. ${ }^{18}$ Conversely, in a prospective study, the Netherlands Study of Depression and Anxiety (NESDA), conducted with 1,209 participants, $66 \%$ women, physical abuse was associated with depression and MADD; however, sexual abuse was not associated with mental disorders. ${ }^{16}$ On the other hand, another study found an association between sexual abuse and MADD. ${ }^{17}$ The differences between the studies could be due to the different definitions of physical and sexual abuse and the different criteria used to diagnose depression and anxiety. However, despite some conflicting results, the association between physical and sexual abuse and mental disorders is consistent. In this respect, the present findings confirm the results of earlier studies, indicating a significant association between physical and sexual abuse and anxiety, depression and MADD.

Another factor found to be independently associated with MADD in the present cohort was smoking. Studies have shown an association between smoking and anxiety and depression in patients with chronic diseases. ${ }^{31,32}$ In the cross-sectional Nord-Trøndelag Health Study (HUNT-2), conducted with 60,814 participants, a significant association was found between smoking and MADD (OR=1.82; 95\%CI: $1.69-1.95){ }^{33}$ Some authors investigated the effect of nicotine on the central nervous system. ${ }^{34}$ In animal models, exposure to nicotine in adolescence was associated with anxiety- and depression-like behaviors due to the persistent state of hyperactive dopamine activity in the ventral tegmental area concomitant with hyperactive neuronal activity states in the prefrontal cortex. ${ }^{34}$ In addition, smoking could be associated with MADD because this behavior is not very socially acceptable in Brazil, negatively affecting women's self-esteem, as has already been reported in a different cultural setting. ${ }^{32}$

Some limitations of the present study should be taken into consideration when interpreting its findings. The cross-sectional design does not permit any causal inferences to be made. Another limitation refers to the lack of data on the use of medication for mental disorders. The cause of CPP was not investigated; however, evidence suggests that mental disorders in women with CPP are more likely to reflect the effects of chronic pain rather than being the cause of that pain. ${ }^{35}$

The strongpoints of this study include the existence of a control group of pain-free women and the use of a covariate-adjusted regression model. To the best of our knowledge, this is the first study to show an association between CPP and MADD. Furthermore, the use of the HADS is important, since this is a validated instrument widely used in health research, including in patients with chronic pain. Another important point that should be mentioned is the identification of associations between sociodemographic, behavioral and clinical factors and anxiety, depression and MADD.

The findings of the present study suggest that systematic management of psychological factors could contribute towards improving the mental health of women with CPP. Therefore, biopsychosocial and interdisciplinary management is recommended.

\section{Conclusion}

In conclusion, anxiety, depression and MADD were more prevalent in women with CPP compared to those without CPP. A significant association was found between pelvic pain, physical abuse, sexual abuse and smoking and mental disorders. The independent association between CPP and anxiety, depression and MADD was particularly noteworthy.

\section{Author contributions}

All authors contributed to data analysis, drafting or revising the article, gave final approval of the version to be published, and agree to be accountable for all aspects of the work.

\section{Disclosure}

The authors report no conflicts of interest in this work.

\section{References}

1. Bahouq H, Allali F, Rkain H, Hajjaj-Hassouni N. Discussing sexual concerns with chronic low back pain patients: barriers and patients' expectations. Clin Rheumatol. 2013;32(10):1487-1492. doi:10.1007/ s10067-013-2299-y

2. Howard FM. The role of laparoscopy in the chronic pelvic pain patient. Clin Obstet Gynecol. 2003;46(4):749-766.

3. Coelho LS, Brito LM, Chein MB, et al. Prevalence and conditions associated with chronic pelvic pain in women from São Luís, Brazil. Braz J Med Biol Res. 2014;47(9):818-825. 
4. Lou WJ, Chen B, Zhu L, et al. Prevalence and factors associated with female sexual dysfunction in Beijing, China. Chin Med J. 2017;130 (12):1389-1394. doi:10.4103/0366-6999.207466

5. Choung RS, Herrick LM, Locke GR 3rd, Zinsmeister AR, Talley NJ. Irritable bowel syndrome and chronic pelvic pain: a population-based study. J Clin Gastroenterol. 2010;44(10):696-701. doi:10.1097/ MCG.0b013e3181d7a368

6. Ayorinde AA, Bhattacharya S, Druce KL, Jones GT, Macfarlane GJ. Chronic pelvic pain in women of reproductive and post-reproductive age: a population-based study. Eur J Pain. 2017;21(3):445-455. doi:10.1002/ejp.938

7. Zondervan KT, Yudkin PL, Vessey MP, et al. Chronic pelvic pain in the community: symptoms, investigations, and diagnoses. Am J Obstet Gynecol. 2001;184(6):1149-1155. doi:10.1067/mob.2001.112904

8. Warren JW, Morozov V, Howard FM. Could chronic pelvic pain be a functional somatic syndrome? Am J Obstet Gynecol. 2011;205 (3):199.e1-199.e5. doi:10.1016/j.ajog.2011.04.003

9. Kaya B, Unal S, Ozenli Y, Gursoy N, Tekiner S, Kafkasli A. Anxiety, depression and sexual dysfunction in women with chronic pelvic pain. Sex Relation Ther. 2006;21(2):187-196. doi:10.1080/ 14681990500359897

10. Romão AP, Gorayeb R, Romão GS, et al. High levels of anxiety and depression have a negative effect on quality of life of women with chronic pelvic pain. Int J Clin Pract. 2009;63(5):707-711. doi:10.1111/j.1742-1241.2009.02034.x

11. Da Luz RA, de Deus JM, Conde DM. Quality of life and associated factors in Brazilian women with chronic pelvic pain. J Pain Res. 2018;11:1367-1374. doi:10.2147/JPR.S168402

12. Latthe P, Mignini L, Gray R, Hills R, Khan K. Factors predisposing women to chronic pelvic pain: systematic review. BMJ. 2006;332 (7544):749-755. doi:10.1136/bmj.38748.697465.55

13. Miller-Matero LR, Saulino C, Clark S, Bugenski M, Eshelman A, Eisenstein D. When treating the pain is not enough: a multidisciplinary approach for chronic pelvic pain. Arch Womens Ment Health. 2016;19(2):349-354. doi:10.1007/s00737-015-0537-9

14. As-Sanie S, Clevenger LA, Geisser ME, Williams DA, Roth RS. History of abuse and its relationship to pain experience and depression in women with chronic pelvic pain. Am J Obstet Gynecol. 2014;210(4):317.e1-317.e8. doi:10.1016/j.ajog.2013.12.048

15. Lindert J, von Ehrenstein OS, Grashow R, Gal G, Braehler E, Weisskopf MG. Sexual and physical abuse in childhood is associated with depression and anxiety over the life course: systematic review and meta-analysis. Int $J$ Public Health. 2014;59(2):359-372. doi: 10.1007/s00038-013-0519-5

16. Hovens JG, Giltay EJ, Wiersma JE, Spinhoven P, Penninx BW, Zitman FG. Impact of childhood life events and trauma on the course of depression and anxiety disorders. Acta Psychiatr Scand. 2012;126 (3):198-207. doi:10.1111/j.1600-0447.2011.01828.x

17. Chou KL. Childhood sexual abuse and psychiatric disorders in middle-aged and older adults: evidence from the 2007 adult psychiatric morbidity survey. J Clin Psychiatry. 2012;73(11):e1365-e1371. doi:10.4088/JCP.12m07946

18. Tarzia L, Maxwell S, Valpied J, Novy K, Quake R, Hegarty K. Sexual violence associated with poor mental health in women attending Australian general practices. Aust N Z J Public Health. 2017;41 (5):518-523. doi:10.1111/1753-6405.12685

19. Zigmond AS, Snaith RP. The hospital anxiety and depression scale. Acta Psychiatr Scand. 1983;67(6):361-370.
20. Botega NJ, Pondé MP, Medeiros P, Lima MG, Guerreiro CA. Validation of the hospital anxiety and depression scale in ambulatory epileptic patients. J Bras Psiquiatr. 1998;47(6):285-289.

21. Skov T, Deddens J, Petersen M, Endahl L. Prevalence proportion ratios: estimation and hypothesis testing. Int J Epidemiol. 1998;27 (1):91-95.

22. World Health Organization. Depression and Other Common Mental Disorders. Global Health Estimates. Geneva: World Health Organization; 2017.

23. Takamatsu K, Fujii E, Ohta H. The impact of benign gynecological diseases on mental health. J JSPOG. 2002;7(2):247-254.

24. Shen TC, Yang CY, Huang YJ, Lin CL, Sung FC. Risk of depression in patients with uterine leiomyoma: a nationwide population-based cohort study. J Affect Disord. 2017;213:126-130. doi:10.1016/j. jad.2017.02.020

25. Luz RA, Rodrigues FM, Vila VS, Deus JM, Lima KP. Depressive symptoms in women with chronic pelvic pain. Rev Bras Ginecol Obstet. 2014;36(2):79-83.

26. Baxter AJ, Scott KM, Ferrari AJ, Norman RE, Vos T, Whiteford HA. Challenging the myth of an "epidemic" of common mental disorders: trends in the global prevalence of anxiety and depression between 1990 and 2010. Depress Anxiety. 2014;31(6):506-516. doi:10.1002/ da. 22230

27. Miller LR, Cano A. Comorbid chronic pain and depression: who is at risk? J Pain. 2009;10(6):619-627. doi:10.1016/j.jpain.2008. 12.007

28. Arnow BA, Hunkeler EM, Blasey CM, et al. Comorbid depression, chronic pain, and disability in primary care. Psychosom Med. 2006;68(2):262-268. doi:10.1097/01.psy.0000204851.15499.fc

29. Baxter AJ, Scott KM, Vos T, Whiteford HA. Global prevalence of anxiety disorders: a systematic review and meta-regression. Psychol Med. 2013;43(5):897-910. doi:10.1017/S003329171200 147X

30. Flores RJ, Campo-Arias A, Stimpson JP, Chalela CM, Reyes-Ortiz CA. The association between past sexual abuse and depression in older adults from Colombia. J Geriatr Psychiatry Neurol. 2018;31 (1):13-18. doi:10.1177/0891988717743588

31. Hamrah MS, Hamrah MH, Ishii H, et al. Anxiety and depression among hypertensive outpatients in Afghanistan: a cross-sectional study in Andkhoy city. Int $J$ Hypertens. 2018;2018:8560835. Available from: https://www.hindawi.com/journals/ijhy/2018/ 8560835/. Accessed November 06, 2018.

32. Liu Q, Cai H, Yang LH, et al. Depressive symptoms and their association with social determinants and chronic diseases in middleaged and elderly Chinese people. Sci Rep. 2018;8(1):3841. doi:10.1038/s41598-018-22175-2

33. Mykletun A, Overland S, Aarø LE, Liabø HM, Stewart R. Smoking in relation to anxiety and depression: evidence from a large population survey: the HUNT study. Eur Psychiatry. 2008;23(2):77-84. doi:10.1016/j.eurpsy.2007.10.005

34. Jobson CL, Renard J, Szkudlarek H, et al. Adolescent nicotine exposure induces dysregulation of mesocorticolimbic activity states and depressive and anxiety-like prefrontal cortical molecular phenotypes persisting into adulthood. Cereb Cortex. 2018. doi:10.1093/ cercor/bhy 179

35. Roth RS, Punch M, Bachman JE. Psychological factors in chronic pelvic pain due to endometriosis: a comparative study. Gynecol Obstet Invest. 2011;72(1):15-19. doi:10.1159/000321392 


\section{Publish your work in this journal}

The Journal of Pain Research is an international, peer reviewed, open access, online journal that welcomes laboratory and clinical findings in the fields of pain research and the prevention and management of pain Original research, reviews, symposium reports, hypothesis formation and commentaries are all considered for publication. The manuscript management system is completely online and includes a very quick and fair peer-review system, which is all easy to use. Visit http:// www.dovepress.com/testimonials.php to read real quotes from published authors.

Submit your manuscript here: https://www.dovepress.com/journal-of-pain-research-journal 